\title{
Cutaneous Metastasis as the First Presentation of Non-Small-Cell Lung Cancer with a BRAF Mutation: A Case Report
}

This article was published in the following Dove Press journal: OncoTargets and Therapy

\author{
Xuejun Wang ${ }^{1, *}$ \\ Hongmei Wangl'* \\ Baochang Jia' \\ Fang $\mathrm{He}^{2}$ \\ Yawei Yuan' \\ Weijun Zhang' \\ 'Department of Radiation Oncology, \\ Affiliated Cancer Hospital \& Institute of \\ Guangzhou Medical University, \\ Guangzhou, Guangdong Province, \\ People's Republic of China; ${ }^{2}$ Department \\ of Radiation Oncology, Zhongshan City \\ People's Hospital, Zhongshan, Guangdong \\ Province, People's Republic of China
}

*These authors contributed equally to this work
Correspondence: Weijun Zhang; Hongmei Wang

Department of Radiation Oncology,

Affiliated Cancer Hospital \& Institute of

Guangzhou Medical University,

Guangzhou, Guangdong Province,

People's Republic of China

Tel +86 I3688879087

Email591533086@qq.com;

hongmeiWI988@I63.com

\begin{abstract}
Cutaneous metastasis from a primary visceral malignancy is a relatively uncommon clinical manifestation that occurs as an initial presentation in $1 \%$ to $12 \%$ of patients with internal malignancies. Additionally, cutaneous metastases are often late signs of an internal malignancy, and in very rare cases they may occur at the same time or before the primary cancer has been detected. Metastasis to the skin has a poor prognosis and is often a sign of widespread malignant tumors. In the present study, we report a 72-year-old male who presented with multiple rapidly growing subcutaneous nodules. Positron emission tomography-computed tomography (PET-CT) revealed a hypermetabolic concentration of radiotracer in the left lower lung and multiple organ metastases associated with multiple skin masses. Biopsy of one of the skin nodules and gene detection indicated metastatic adenocarcinoma consistent with a primary lung origin with a BRAF mutation. BRAF mutations are emerging therapeutic targets in non-small-cell lung cancer (NSCLC), as they are present in $2-4 \%$ of NSCLC cases. To the best of our knowledge, this is the first case report to show that BRAF-mutant lung adenocarcinoma can be associated with cutaneous metastasis. Early diagnosis and individualized treatment strategies may prolong patient survival.
\end{abstract}

Keywords: metastasis, skin lesion, lung adenocarcinoma, BRAF mutation

\section{Introduction}

Distant metastases at the time of non-small-cell lung cancer (NSCLC) presentation are a frequent clinical problem. ${ }^{1,2}$ The most common sites of metastases from lung cancer are the liver, bones, brain, adrenal glands, lymph nodes, and, rarely, skin. ${ }^{1}$ In a review of 7316 cancer patients, skin involvement as a presenting sign was seen in only $0.8 \%$ of patients. ${ }^{2}$ Generally, cutaneous metastasis develops after the initial diagnosis of the primary internal malignancy and often late in the course of the disease; however, this can be the first presenting sign in very rare cases. ${ }^{3-5}$ The frequencies of the different types of metastases are usually related to the frequency of the relative primary cancer, which varies according to age and sex. ${ }^{6,7}$ The incidence of cutaneous metastasis increases with age and more so after the fifth decade of life. ${ }^{4}$ Furthermore, the most common primary sites for males and females are the lungs and breast, respectively. ${ }^{6,8}$ The presentation of cutaneous metastasis in lung cancer portends a poor prognosis, with an average survival ranging between three and six months in the majority of studies. ${ }^{2,8,9}$ The presence of a firm, rapidly growing papular or nodular lesion should prompt the clinician to suspect internal 
malignancy. In our report, we describe an unusual case of BRAF-mutant lung adenocarcinoma that metastasized to the skin with multiple organ metastases at the time of initial diagnosis. At present, the disease is stable more than 10 months after targeted therapy.

\section{Case Presentation}

A 72-year-old Chinese male was admitted to our hospital in October 2019 with complaints of repeated cough and white sputum for 4 months. He also discovered multiple painless subcutaneous nodules rapidly growing throughout the body for nearly 3 months. His past medical history included chronic obstructive airway disease (COPD) and an approximately 50-year smoking history (one pack/day). He had no other important medical history, and the family had no history of cancer. After the skin nodules were found, the patient experienced no discomfort, and no treatment was sought until the lesions had increased in size. On admission, his pulse rate was 85 beats per minute, respiratory rate was 19 breaths per minute, blood pressure was $128 / 75 \mathrm{~mm} \mathrm{Hg}$, and oxygen saturation was 99\% on room air.

Upon physical examination, nodules were found in multiple subcutaneous areas (bilateral upper arm, face, back of chest, abdominal wall, and left armpit). On palpation, the size of the nodules was found to be approximately $0.5-2 \mathrm{~cm}$ in diameter. None of the nodules demonstrated bleeding or exudation. The largest subcutaneous nodule, located in the left chest wall, presented with a hard texture, smooth surface, and poor mobility and was subsequently biopsied. No obvious abnormalities were found from other physical examinations.

The laboratory examinations revealed elevated white blood cells $\left(12.31 \times 10^{\wedge} 9 / \mathrm{L}\right.$; normal range, 3.5-9.5 $\times 10^{\wedge} 9 /$ L), neutrophil percentage (77.6\%; normal range, 40-75\%), cytokeratin 19 fragment (CYFRA 21-1) concentration (21.88 ng/mL; normal range, 0-3.3 ng/mL), neuronspecific enolization (enolase) enzyme (NSE; $158.80 \mathrm{ng} /$ $\mathrm{mL}$; normal range, $0-16.3 \mathrm{ng} / \mathrm{mL}$ ) and tumor-specific growth factor (TSGF; $68 \mathrm{U} / \mathrm{mL}$; normal range, 0-64 U/ $\mathrm{mL}$ ). The results of other laboratory tests, such as liver, kidney, and coagulation profiles, were within normal ranges. After admission, the whole-body PET-CT showed intense uptake of the radiotracer fluorine-18 fluorodeoxyglucose by the left and right lungs, revealing multiple metastases (Figure 1A). The largest subcutaneous metastatic nodule was located in the left chest wall. The PETCT scan identified unpalpable nodules that could have been easily missed. In addition, hypermetabolic lesions were observed in the thyroid (Figure 1B), adrenal gland (Figure 1C), bone (Figure 1D) and left psoas (Figure 1E). However, no sign of high metabolic activity in the brain was found, indicating that metastasis to the brain had not occurred.

We took pictures of subcutaneous nodules in the chest and abdomen of the patient (Figure 2A). At the same time, a biopsy was performed on the largest subcutaneous nodule of the left chest wall. The histology showed that the malignant tumor consisted of an adenoid pattern with some necrosis. Immunohistochemistry was performed in our hospital on samples taken from the largest subcutaneous nodules in our hospital, showing that the tumor cells were strongly positive for CK7, TTF-1 and Napsin A (Figure 2B-F) and negative for CK20 and P63. The results indicated metastatic adenocarcinoma of lung origin. Additionally, a positive mutation in the BRAF gene (D594G) was detected following sequencing of the tissue samples. The tests for EGFR and ALK mutation were negative. Finally, this patient was considered to have lung adenocarcinoma, cT4N3M1c, stage IVB. Given the diagnosis of stage IV lung adenocarcinoma with multiple distant metastases, the patient was considered unsuitable for surgery; he also refused systemic chemotherapy. Although the relationship between this mutation and the efficacy of BRAF inhibitors is currently unclear, the patient was advised to start molecular targeted therapy. He was treated with vemurafenib $480 \mathrm{mg}$ daily by oral administration and continues to take the medication. Additionally, regular bone phosphorus acupuncture treatment was performed. The sizes of the subcutaneous nodules significantly decreased after one month of treatment. According to PET-CT, the largest tissue mass at the time of writing was approximately $2 \mathrm{~cm} \times 1.4 \mathrm{~cm} \times 1.7 \mathrm{~cm}$, which was located in the lower left lung (Figure 3A), and multiple hilar lymph nodes and flocculent shadows remained scattered in the left and right lungs. The size of the mass in the left lung slightly decreased according to the lung computed tomography (CT) performed in December 2019 (Figure 3B) and March 2020 (Figure 3C). During the follow-up period, the patient developed fatigue and scattered rashes on his chest after taking the medicine for one month. The pulmonary inflammation was further aggravated for nearly six months. Grade $\geq 3$ treatment-related AEs have not yet been reported. To date, the patient's general condition has remained stable. 

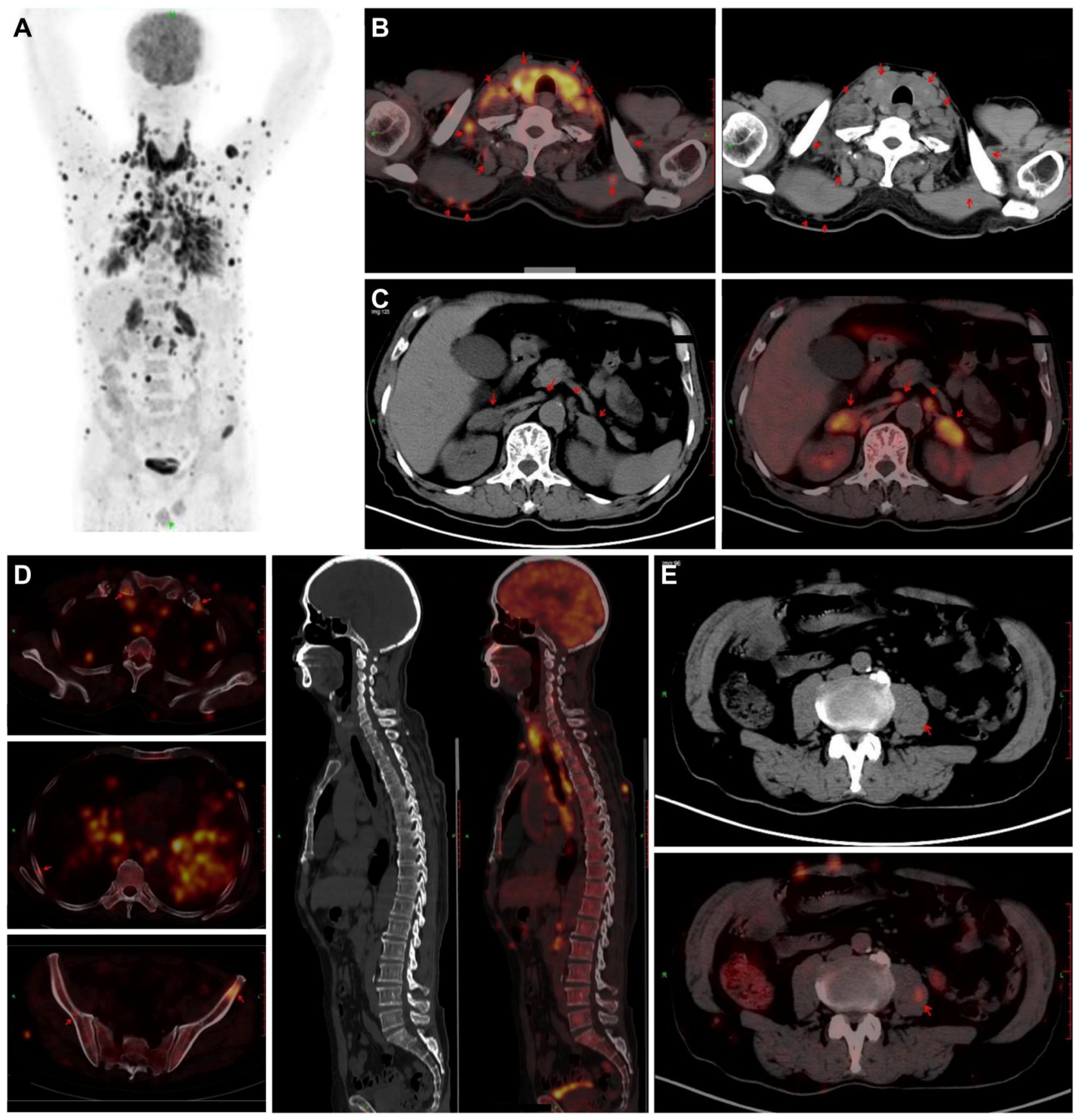

Figure I Whole-body PET-CT of primary lung cancer.

Notes: (A) Whole-body PET-CT showing multiple lymph nodes and cutaneous metastasis. The largest subcutaneous metastatic nodule was located in the left chest wall. (B-E) Whole-body scans of, in order, the thyroid, adrenal glands, skull and buttocks. Hypermetabolic activity was observed in these areas.

Abbreviation: PET-CT, positron emission tomography-computed tomography.

\section{Ethics Approval and Consent for Publication}

This study was approved by the research ethics committee of the Affiliated Cancer Hospital of Guangzhou Medical University, and written informed consent for publication of the clinical details and images from the study was obtained from the patient and his daughter.

\section{Discussion}

Based on a large-scale investigation of patients with NSCLC, the incidence of distant metastasis at the time of initial diagnosis is $47.3 \% .^{1}$ Skin metastases are encountered in $0.7-9 \%$ of all patients with cancer, although compared with other organs, the skin is an uncommon site of metastatic disease. ${ }^{10}$ Dissemination of visceral 


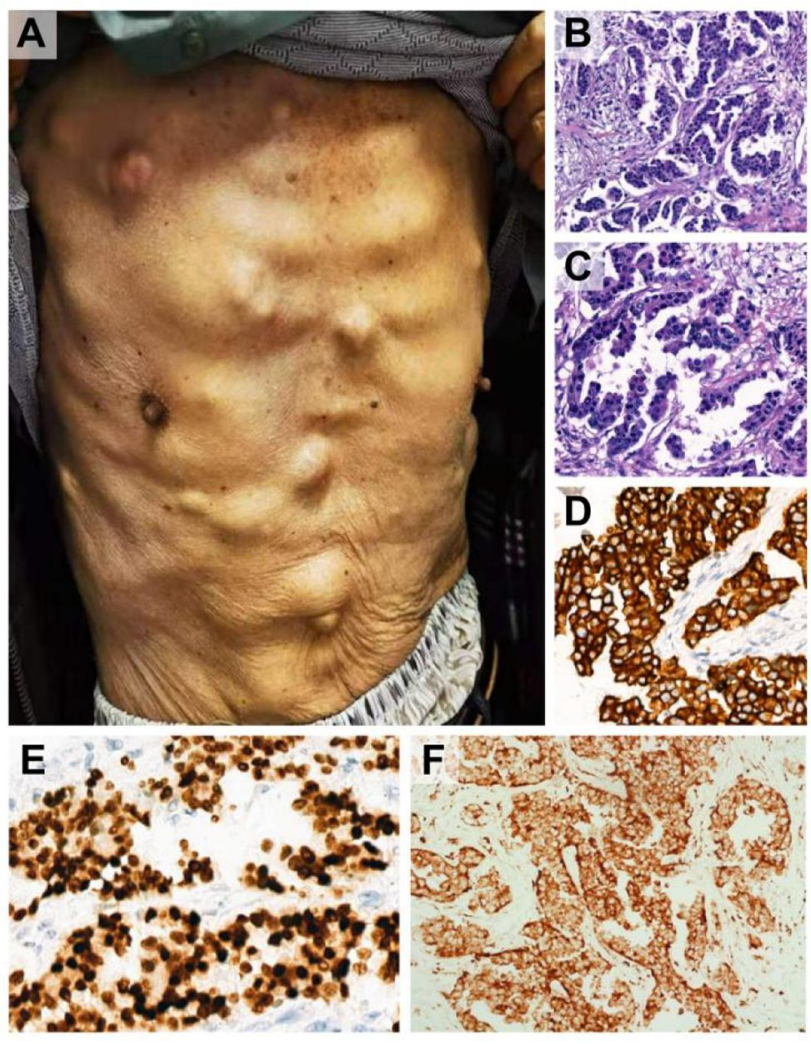

Figure 2 Cutaneous metastasis of lung adenocarcinoma. (A) Subcutaneous nodules in the chest and abdomen. Skin metastasis biopsy from the left chest wall shows poorly differentiated metastatic adenocarcinoma (H\&E, original magnification $\times 100$ (B), $\times 200$ (C)). (D-F) Immunohistochemical staining shows positivity for, in order, CK7 (original magnification $\times 200$ ), TTF-I (original magnification $\times 200$ ) and napsin A (original magnification $\times 200$ ), indicating that the metastasis is of lung origin. Abbreviations: H\&E, hematoxylin and eosin; CK7, cytokeratin 7; TTF-I, thyroid transcription factor I.

malignancies to the skin usually occurs in later stages of the disease. However, cutaneous metastases may be the first indication of clinically silent visceral malignancies. The most common sites of metastases from lung cancer are the liver, bones, brain, adrenal glands, lymph nodes, and, rarely, skin. ${ }^{1,3,7}$ The source of the primary tumor responsible for cutaneous deposits differs between males and females. In men, malignancies that most often metastasize to the skin are located in the lung, large intestine, oral cavity, kidney, breast, esophagus, pancreas, stomach, and liver. In contrast, in women the most common origins of skin metastases are the breast, ovary, oral cavity, lung, and large intestine. ${ }^{11}$ According to the current research, the relationship between the histological type of lung cancer and the occurrence of cutaneous metastasis has not been well defined. In one study, adenocarcinomas gave rise to cutaneous metastases at a higher frequency than other histologic subtypes. ${ }^{12}$ In contrast, another study showed that large-cell carcinoma was associated with a tendency to disseminate to the skin. ${ }^{13}$

The clinical presentations of skin metastases are often highly variable, nonspecific, and subtle and are easily mistaken for other dermatological conditions. ${ }^{10,11}$ Skin metastases are a sign of an aggressive, poorly differentiated cancer. Those that arise from lung cancer may develop before the primary tumor is recognized and can be spread by the venous, arterial, or lymphatic system. The anterior chest and the head and neck are the most common sites of metastatic lesions for men, whereas women tend to present with metastases at the abdomen and anterior chest wall. ${ }^{7}$ Cutaneous metastases may present with different clinical features; typical features include painless nodules of firm or elastic consistency that develop within a short time. ${ }^{8,9}$ These lesions can be single or multiple and commonly appear as a dermal nodule, sometimes ulcerated; however, several other presentations can occur. ${ }^{6}$ The size of the skin lesions is very variable and can fluctuate from $5 \mathrm{~mm}$ to $6 \mathrm{~cm}$ diameter. ${ }^{14}$ Asymptomatic elderly patients presenting with multiple, painless, immobile cutaneous nodules should be considered for further investigation to determine the underlying primary cause. ${ }^{15}$ The cutaneous
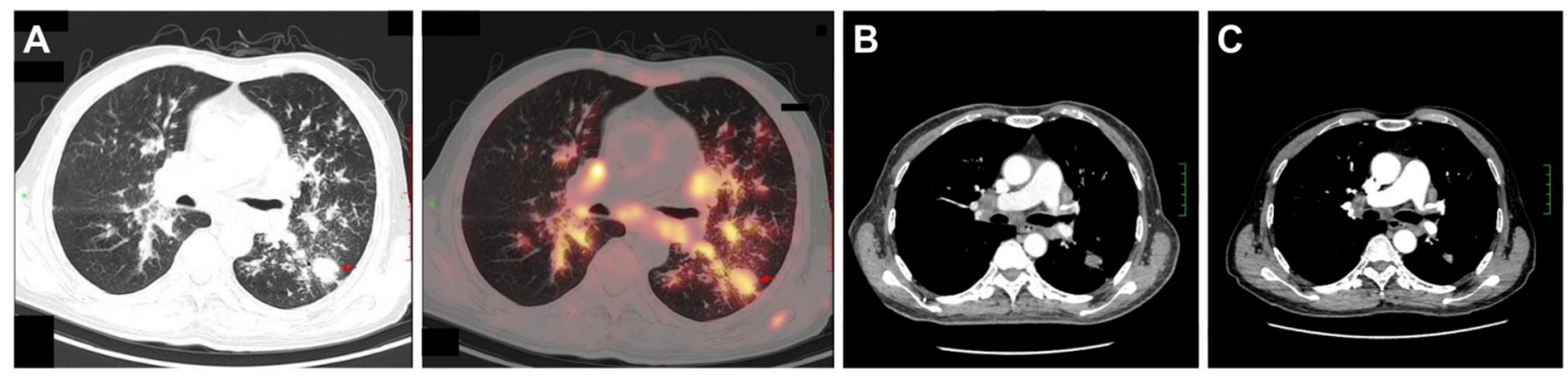

Figure 3 Imaging of lung cancer. (A) PET-CT indicates that the largest tissue mass is in the lower left lung. During treatment, additional CT scans were performed in (B) December 2019 and (C) March 2020.

Abbreviations: PET-CT, positron emission tomography-computed tomography; CT, computed tomography. 
metastasis observed in our case consisted of multiple nodules that were immobile, hard, and painless.

Cutaneous metastases may be unrecognized in the clinic, as they may be mistaken for certain dermatological conditions. A high index of clinical suspicion is required for an early diagnosis to enable prompt treatment. ${ }^{5,7}$ The histopathological features of metastases are occasionally difficult to distinguish from those of primary cutaneous tumors despite the rigorous application of useful techniques, particularly immunohistochemistry. ${ }^{16}$ Biopsy remains the gold standard for the initial diagnosis and confirmation of the diagnosis of cutaneous metastasis. ${ }^{15}$ Adenocarcinoma is the most common type of metastasis from lung cancer, followed by squamous cell carcinoma (SCC). ${ }^{7}$ Immunohistochemical markers are useful for the identification of the primary cancer responsible for skin metastasis. To establish the origin of an adenocarcinoma from a pulmonary primary site, a combination of CK7, CK20, Naspina and TTF-1 markers should be used. CK7 is the usual molecular species of keratin detected in primary lung adenocarcinoma. TTF-1 expression is prominent in patients with cancers of pulmonary and thyroid carcinoma origin. Anti-TTF is both specific and sensitive for primary adenocarcinoma, whereas CK7+ and CK20- are sensitive but not specific. ${ }^{4,10}$ In our case, strong and diffuse nuclear expression of TTF-1 and CK7 immunostaining was characteristic of primary lung cancer, which was helpful in identifying the histological origin of the primary cancer. Biopsy of the skin nodules combined with immunohistochemistry led to the diagnosis of skin metastasis from lung adenocarcinoma. In addition, PET-CT scans can be used as an effective tool for diagnosis and evaluation. ${ }^{17}$ In our case, PET-CT implied the existence of metastasis despite the lack of positive findings on physical examination. Genetic testing was performed on November 7, 2019, from a sample taken from a skin nodule and revealed a BRAF D594G mutation. This is the first case report to show that BRAF-mutant lung adenocarcinoma can be associated with cutaneous metastasis.

Cutaneous metastasis in visceral cancers portends a poor prognosis and is often a signal of a widely spread malignancy. ${ }^{18}$ Such malignancies are usually associated with an advanced neoplastic process with metastasis to other organs. ${ }^{5}$ Unfortunately, half of the patients with cutaneous metastasis die within the first 6 months after the diagnosis. ${ }^{9}$ Treatment of cutaneous metastasis emphasizes primary cancer therapy, and combinations of chemotherapy and radiation therapy tend to be applied in clinical practice. $^{15}$ If multiple lesions are present, chemotherapy alone is an option but may cause an inadequate response. Radiation therapy can be used if the chemotherapy is associated with severe pain or bleeding. ${ }^{7,14}$ The patient in our case had a BRAF gene mutation; these mutations occur in $1-4 \%$ of NSCLC patients. ${ }^{19,20}$ Non-V600E mutations are more likely to be observed in smokers than V600E mutations, which is consistent with previously reported results. $^{21,22}$ The value of BRAF mutations, especially of non-V600E mutations, in predicting the efficiency of targeted agents remains unclear. No significant differences in age, histology type, performance status, or stage at diagnosis between V600E and non-V600E mutations have yet been found. ${ }^{22}$ BRAF mutations can be distinguished by their kinase activity and signaling mechanism via the mitogen-activated protein kinase (MAPK) pathway into three classes: V600-mutant kinase-activating monomers (class I), kinase-activating dimers (class II), and kinase-inactivating heterodimers (class III). D594G belongs to class III with impaired kinase activity but increased MAPK pathway signaling. Compared with class I, classes II and III have distinct clinical and molecular features, including poorer clinical outcomes. $^{23,24}$ Targeted therapies are currently only approved for V600E mutations; much less is known about non-V600E mutations. ${ }^{19,21}$ A multiple nonmelanoma basket trial reported a response rate of $42 \%$ and a median progression-free survival (PFS) of 7.3 months in the 19 NSCLC patients with BRAFV600-mutated tumours treated with vemurafenib. ${ }^{25}$ In addition, an ORR of $33 \%$ and PFS of 5.5 months have been reported for dabrafenib used as monotherapy. ${ }^{26}$ One study evaluated the efficacy of combinatorial treatment comprising a BRAF inhibitor, dabrafenib, and an MEK inhibitor, trametinib, and reported an ORR of $63 \%$ and PFS of 9.7 months. $^{27}$ The European Society for Medical Oncology (ESMO) guidelines recommend dabrafenib combined with trametinib for BRAF inhibitor-naive patients with BRAF V600-mutant NSCLC (stage IV). ${ }^{28}$ Preclinical data suggest that the NSCLC tumors of some BRAF non-V600 mutants may be sensitive to dabrafenib combined with trametinib but less sensitive to dabrafenib alone. $^{29}$ In a descriptive study, 31 eligible patients with stage IIIC or IV melanoma and non-V600E BRAF mutations received vemurafenib $(960 \mathrm{mg}$, twice daily). Responses were observed in patients with V600K $(n=3), \operatorname{V600E2}(n=1), \operatorname{V600R}(n=1)$, L597S $(n=1)$ and D594G ( $=1)$ mutations. No new safety signals were reported. Vemurafenib showed activity in patients with advanced melanoma with rarer BRAF mutations. ${ }^{30}$ The recent AcSé study demonstrated that vemurafenib 
monotherapy is effective for BRAF V600-mutated NSCLC but not for BRAF non-V600-mutant NSCLC. ${ }^{21}$ However, the AcSé study did not include the mutation type of the patients. In our case, the patient was treated with vemurafenib-based targeted therapy. The survival time of the patient has now exceeded the average survival time.

\section{Conclusion}

We have reported the first case of BRAF-mutant lung adenocarcinoma with multiple cutaneous metastases. Patients with both skin lesions and an extensive history of smoking, especially middle-aged and elderly people who exhibit multiple, painless and inactive skin nodules in a short period of time, should be examined carefully. The value of BRAF mutations, especially of non-V600E mutations, in predicting the efficiency of targeted agents remains unclear. Research needs to focus on therapies, including combination therapies, that specifically target BRAF non-V600 mutations. Personalized therapy may be necessary to effectively target these molecular subsets.

\section{Abbreviations}

PET-CT, positron emission tomography-computed tomography; NSCLC, non-small-cell lung cancer; COPD, chronic obstructive airway disease; NSE, neuron-specific enolization; TSGF, tumor-specific growth factor; H\&E, hematoxylin and eosin; CK7, cytokeratin 7; TTF-1, thyroid transcription factor 1 ; CT, computed tomography.

\section{Ethics Approval and Informed Consent}

All procedures performed in studies involving human participants conformed to the standards set by the Declaration of Helsinki and were approved by the Human Ethics Committee of the Affiliated Cancer Hospital \& Institute of Guangzhou Medical University.

\section{Consent for Publication}

The patient and his daughter provided a written informed consent form and agreed to publish the details of the case.

\section{Acknowledgments}

This paper was supported by the Guangzhou Key Medical Discipline Construction Project.

\section{Funding}

No funding was provided for the production of this case report.

\section{Disclosure}

The authors declare that they have no conflicts of interest.

\section{References}

1. Tamura T, Kurishima K, Nakazawa K, et al. Specific organ metastases and survival in metastatic non-small-cell lung cancer. Mol Clin Oncol. 2015;3(1):217-221. doi:10.3892/mco.2014.410

2. Lookingbill DP, Spangler N, Sexton FM. Skin involvement as the presenting sign of internal carcinoma. A retrospective study of 7316 cancer patients. J Am Acad Dermatol. 1990;22(1):19-26. doi:10. 1016/0190-9622(90)70002-Y

3. Liao H, Wu S, Karbowitz SR, Morgenstern N, Rose DR. Cutaneous metastasis as an initial presentation of lung adenocarcinoma with KRAS mutation: a case report and literature review. Stem Cell Investig. 2014;1:6. doi:10.3978/j.issn.2306-9759.2014.03.04

4. Saeed S, Keehn CA, Morgan MB. Cutaneous metastasis: a clinical, pathological, and immunohistochemical appraisal. J Cutan Pathol. 2004;31(6):419-430. doi:10.1111/j.0303-6987.2004.00207.x

5. McSweeney WT, Tan K. Cutaneous metastases as a presenting sign of metastatic NSCLC. J Surg Case Rep. 2019;2019(10):rjz279. doi:10.1093/jscr/rjz279

6. Guanziroli E, Coggi A, Venegoni L, et al. Cutaneous metastases of internal malignancies: an experience from a single institution. Eur J Dermatol. 2017;27(6):609-614. doi:10.1684/ejd.2017.3142

7. Khaja M, Mundt D, Dudekula RA, et al. Lung cancer presenting as skin metastasis of the back and hand: a case series and literature review. Case Rep Oncol. 2019;12(2):480-487. doi:10.1159/000501 363

8. Krathen RA, Orengo IF, Rosen T. Cutaneous metastasis: a meta-analysis of data. South Med J. 2003;96(2):164-167. doi:10.1097/01.SMJ.0000053676.73249.E5

9. Song Z, Lin B, Shao L, Zhang Y. Cutaneous metastasis as a initial presentation in advanced non-small cell lung cancer and its poor survival prognosis. $J$ Cancer Res Clin Oncol. 2012;138 (10):1613-1617. doi:10.1007/s00432-012-1239-6

10. Hussein MR. Skin metastasis: a pathologist's perspective. J Cutan Pathol. 2010;37(9):e1-e20. doi:10.1111/j.1600-0560.2009.01469.x

11. Queirós CS, Filipe PL, de Almeida LS. Cutaneous metastases from solid neoplasms in the 21st century: a retrospective study from a Portuguese tertiary care center. J Eur Acad Dermatol Venereol. 2020;34(6):1218-1224. doi:10.1111/jdv.16120

12. Hu SC, Chen GS, Wu CS, Chai CY, Chen WT, Lan CC. Rates of cutaneous metastases from different internal malignancies: experience from a Taiwanese medical center. $J$ Am Acad Dermatol. 2009;60(3):379-387. doi:10.1016/j.jaad.2008.10.007

13. Hidaka T, Ishii Y, Kitamura S. Clinical features of skin metastasis from lung cancer. Intern Med. 1996;35(6):459-462. doi:10.2169/ internalmedicine. 35.459

14. Garrido MJM, Ponce CG, Martínez JLS, y Sevila CM, Mena AC, Antón FM. Cutaneous metastases of lung cancer. Clin Transl Oncol. 2006;8(5):330-333. doi:10.1007/s12094-006-0178-6

15. Yu Q, Subedi S, Tong Y, et al. Scalp metastases as first presentation of pulmonary adenocarcinomas: a case report. OncoTargets Ther. 2018;11:6147-6151. doi:10.2147/OTT.S174577

16. Koyuncuer A, Cüzdan SS, Şenköy E, Kalacı N. Skin metastasis as the first manifestation of lung carcinoma. Asian Cardiovasc Thorac Ann. 2018;26(4):320-324. doi:10.1177/0218492318766784 
17. Zhu X, Chen J, Yang F, Tang C. Multiple sites of soft-tissue metastases secondary to lung cancer: a case report. Medicine. 2019;98(49) e18162. doi:10.1097/MD.0000000000018162

18. Alcaraz I, Cerroni L, Rütten A, Kutzner H, Requena L. Cutaneous metastases from internal malignancies: a clinicopathologic and immunohistochemical review. Am J Dermatopathol. 2012;34 (4):347-393. doi:10.1097/DAD.0b013e31823069cf

19. Nguyen-Ngoc T, Bouchaab H, Adjei AA, Peters S. BRAF alterations as therapeutic targets in non-small-cell lung cancer. $J$ Thorac Oncol. 2015;10(10):1396-1403. doi:10.1097/JTO.0000000000000644

20. Baik CS, Myall NJ, Wakelee HA. Targeting BRAF-mutant non-small cell lung cancer: from molecular profiling to rationally designed therapy. Oncologist. 2017;22(7):786-796. doi:10.1634/theoncologist.2016-0458

21. Mazieres J, Cropet C, Montané L, et al. Vemurafenib in non-smallcell lung cancer patients with BRAF(V600) and BRAF(nonV600) mutations. Ann Oncol. 2020;31(2):289-294. doi:10.1016/j. annonc.2019.10.022

22. Tissot C, Couraud S, Tanguy R, Bringuier PP, Girard N, Souquet PJ. Clinical characteristics and outcome of patients with lung cancer harboring BRAF mutations. Lung Cancer. 2016;91:23-28. doi:10.1016/j.lungcan.2015.11.006

23. Dagogo-Jack I, Martinez P, Yeap BY, et al. Impact of BRAF mutation class on disease characteristics and clinical outcomes in BRAF-mutant lung cancer. Clin Cancer Res. 2019;25(1):158-165. doi:10.1158/1078-0432.CCR-18-2062

24. Dankner M, Rose AAN, Rajkumar S, Siegel PM, Watson IR. Classifying BRAF alterations in cancer: new rational therapeutic strategies for actionable mutations. Oncogene. 2018;37(24):3183-3199. doi:10.1038/s41388018-0171-X
25. Hyman DM, Puzanov I, Subbiah V, et al. Vemurafenib in multiple nonmelanoma cancers with BRAF V600 mutations. $N$ Engl J Med. 2015;373(8):726-736. doi:10.1056/NEJMoa1502309

26. Planchard D, Kim TM, Mazieres J, et al. Dabrafenib in patients with BRAFV600E-positive advanced non-small-cell lung cancer: a single-arm, multicentre, open-label, Phase 2 trial. Lancet Oncol. 2016;17(5):642-650. doi:10.1016/S1470-2045(16)00077-2

27. Planchard D, Besse B, Groen HJM, et al. Dabrafenib plus trametinib in patients with previously treated BRAF(V600E)-mutant metastatic non-small cell lung cancer: an open-label, multicentre phase 2 trial. Lancet Oncol. 2016;17(7):984-993. doi:10.1016/S1470-2045(16) 30146-2

28. Planchard D, Popat S, Kerr K, et al. Metastatic non-small cell lung cancer: ESMO clinical practice guidelines for diagnosis, treatment and follow-up. Ann Oncol. 2018;29(Supp14):iv192-iv237. doi:10. 1093/annonc/mdy 275

29. Noeparast A, Teugels E, Giron P, et al. Non-V600 BRAF mutations recurrently found in lung cancer predict sensitivity to the combination of trametinib and dabrafenib. Oncotarget. 2017;8 (36):60094-60108. doi:10.18632/oncotarget.11635

30. Hallmeyer S, Gonzalez R, Lawson DH, et al. Vemurafenib treatment for patients with locally advanced, unresectable stage IIIC or metastatic melanoma and activating exon 15 BRAF mutations other than V600E. Melanoma Res. 2017;27(6):585-590. doi:10.1097/CMR.00 00000000000398
OncoTargets and Therapy

\section{Publish your work in this journal}

OncoTargets and Therapy is an international, peer-reviewed, open access journal focusing on the pathological basis of all cancers, potential targets for therapy and treatment protocols employed to improve the management of cancer patients. The journal also focuses on the impact of management programs and new therapeutic

\section{Dovepress}

agents and protocols on patient perspectives such as quality of life, adherence and satisfaction. The manuscript management system is completely online and includes a very quick and fair peer-review system, which is all easy to use. Visit http://www.dovepress.com/ testimonials.php to read real quotes from published authors. 